\title{
Audiovisuales para la práctica instrumental en un escenario flipped classroom
}

\section{Audiovisuals for Instrumental Practice in a Flipped Classroom Setting}

\author{
José Palazón-Herrera \\ jpalazonherrera@um.es \\ Departamento de Didáctica de la Expresión Plástica, Musical y Corporal \\ Universidad de Murcia \\ Murcia, España \\ ORCID: http://orcid.org/0000-0001-8821-8669
}

doi: 10.7203/LEEME.42.13055

Recibido: 13-09-2018 Aceptado: 21-11-2018. Contacto y correspondencia: José Palazón Herrera, Departamento de Expresión Plástica, Musical y Corporal, Facultad de Educación Universidad de Murcia, Campus de Espinardo, 30100 Murcia. España.

\section{Resumen}

Este artículo presenta una experiencia de aula en la que estudiantes de música de Educación Secundaria Obligatoria han trabajado temas de música moderna bajo una metodología de aula invertida (flipped classroom) usando vídeos como recurso fundamental para la práctica instrumental. Los resultados de un análisis descriptivo muestran una valoración muy positiva por parte del alumnado tanto de la metodología utilizada (tareas en clase frente a las explicaciones del profesor en casa; adaptación al ritmo del alumno; interés por la práctica instrumental; mejor preparación para el trabajo grupal) como de los vídeos diseñados (contenidos similares a una clase expositiva; aumento de la confianza para las clases presenciales; motivación de uso; duración; calidad e independencia del alumno con respecto a su profesor). Aunque se trata de un estudio exploratorio, puede afirmarse que el diseño de vídeos y su uso bajo una metodología adecuada pueden repercutir positivamente en la práctica instrumental dentro y fuera del aula.

Palabras clave: recursos audiovisuales, clase invertida, tecnología educativa, música en secundaria.

\begin{abstract}
This article presents a classroom experience in which Secondary School music students have worked on associated with modern music as part of a flipped classroom methodology using videos as a fundamental resource for instrumental practice. The results of a descriptive analysis show that students have a very positive assessment both of the methodology employed (tasks in class vs explanations by the teacher at home; adaptation to the student's rhythm; interest in instrumental practice; better knowledge for group work) and of the videos designed (contents similar to an expository class; increase of confidence for face-to-face classes; motivation of use; duration; quality and independence of students with respect to their teacher). Although it is an exploratory study, preliminary results demonstrate that the design of videos and their use under an appropriate methodology can have a positive effect on instrumental practice inside and outside the classroom.
\end{abstract}

Key words: audiovisual resources, flipped classroom, educational technology, music in secondary school. 


\section{Introducción}

El aprendizaje de un instrumento musical en Educación Secundaria Obligatoria (ESO, en adelante) supone un auténtico reto para el docente. El número excesivo de estudiantes por aula y la falta de materiales enfocados a esta disciplina obligan a adoptar nuevas metodologías y a diseñar recursos propios que sean realmente útiles a este propósito. El enfoque metodológico flipped classroom podría ser un primer paso para poder encarar seriamente este tema y, dentro de esta metodología, el diseño de vídeos pensados específicamente para el aprendizaje instrumental podría dar una respuesta positiva a este problema.

El enfoque metodológico denominado flipped classroom (FC, a partir de ahora) o 'clase invertida' se debe a los profesores de Secundaria Bergmann y Sams. Éstos, en sus inicios entre 2007 y 2008, comenzaron a distribuir entre sus estudiantes vídeos de sus lecciones como apoyo a sus explicaciones (Bergmann y Sams, 2012), experimentando un gran auge entre las metodologías activas en estos últimos años (Chen, Wang, Kinshuk y Chen, 2014). En esta metodología, el alumnado ve vídeos en casa con las explicaciones del docente y en clase se resuelven dudas y se participa en actividades creativas o colaborativas, recibiendo el correspondiente feedback. De esta manera, se aumenta la interacción docente-discente al disminuir el tiempo invertido por el profesorado en impartir las clases presenciales (Seaman y Gaines, 2013), pudiendo dedicar más tiempo a satisfacer las demandas emocionales y de aprendizaje del estudiantado (Goodwin y Miller, 2013).

Un elemento clave del modelo FC consiste en proporcionar una oportunidad para que el alumnado conozca el contenido de aprendizaje antes de que éste se trabaje en clase (Brame, 2016). El modelo pedagógico FC se entiende como una metodología apoyada en la tecnología (Aidinopoulou y Sampson, 2017), siendo el vídeo el recurso más utilizado. Entre las ventajas que aporta al proceso de enseñanza-aprendizaje el visionado de vídeos, podemos citar que los discentes pueden: ver los vídeos cuando y donde quieran y las veces que quieran, aprender a su propio ritmo, pensar dentro y fuera del aula (Kellinger, 2012) y disponer de estos recursos para su uso utilizando diversas estrategias de enseñanza (Seaman y Gaines, 2013). Por ello, esta metodología no sólo implica un cambio de rol del profesorado, sino que implica igualmente realizar un esfuerzo por producir materiales didácticos que sirvan de preparación a las clases presenciales (Turro, Mengod, Morales y Busquets, 2016). El diseño de materiales didácticos creados específicamente para el estudio de la música en el ámbito de Educación Secundaria concretamente para $4^{\circ}$ de ESO- bajo un enfoque metodológico FC puede consultarse en trabajos como los de Calvillo (2014) y Parra y Gutiérrez (2017).

En el modelo FC, el vídeo se viene utilizando como típico material de enseñanza preclase (Long, Logan y Waugh, 2016). La opción de las 'explicaciones en casa' en formato vídeo ofrece a los estudiantes la posibilidad de ver cómo solucionar un problema, proceso que, en muchas ocasiones, puede ser todo un reto en un libro de texto (Mattis, 2015). 
Respecto a la percepción del alumnado sobre el visionado ${ }^{1}$ de vídeos, Long, Logan y Waugh (2016) subrayaron en un estudio que el estudiantado muestra una actitud positiva hacia el aprendizaje viendo vídeos previos a las sesiones de clase. Así, el alumnado reconoce sus ventajas sobre recursos en formato texto, poniendo de relieve que los vídeos debían de ser breves, estar muy accesibles y presentar las menores deficiencias técnicas posibles. Por su parte, Imran (2013) apunta que los discentes consideran que el vídeo facilita la comprensión y el aprendizaje individual cuando se usa como recurso de aprendizaje pre-clase en un escenario de aula invertida.

No obstante, el modelo FC, muy centrado en el uso de vídeos, no está exento de limitaciones. Por un lado, las habilidades que como profesorado desarrollamos cara a cara no siempre se traducen en la producción de un vídeo, por lo que muchos docentes pueden encontrar esta transición entre 'formatos' de enseñanza como un reto profesional. Por otro lado, muchos docentes subrayan que es difícil encontrar vídeos de buena calidad (Herreid y Schiller, 2013), lo que les obliga a plantearse la creación de sus propios recursos adaptados a sus estudiantes, siendo este el punto de partida de este trabajo.

Los objetivos planteados en este trabajo son:

- Conocer la percepción del alumnado sobre la metodología de clase invertida o FC.

- Conocer la valoración del alumnado sobre el vídeo como recurso de aprendizaje para la práctica instrumental.

\section{Desarrollo de la experiencia}

\subsection{Delimitación del problema}

Una de las disciplinas de estudio musical en las que los docentes suelen tener mayores dificultades a la hora de trabajar en el aula es la interpretación instrumental, que es parte fundamental del currículo de música de ESO. El número excesivo de alumnos por aula y la carencia de materiales pensados específicamente para un correcto montaje de las piezas instrumentales - materiales prácticamente inexistentes en los libros de texto- convierten la práctica instrumental en un auténtico reto para el profesorado y para el alumnado.

Por una parte, un alumnado muy numeroso en el aula dificulta enormemente las explicaciones del profesorado en materia de técnica instrumental. Mientras que en una escuela de música o en un conservatorio las clases de técnica instrumental se imparten en grupos muy reducidos de personas, en un instituto este tipo de clases hay que aplicarlo a grupos de 30 estudiantes, siendo prácticamente imposible que el docente haga un seguimiento razonable del alumnado sin invertir muchísimo tiempo en clase.

\footnotetext{
${ }^{1}$ Entiéndase 'visionado' como el proceso de ver un vídeo enfocado a un objetivo educativo: aprender un concepto, ver cómo realizar una acción, etc.
}

@JOSÉ PALAZÓN-HERRERA. THE CONTENT OF THIS ARTICLE IS THE SOLE RESPONSIBILITY OF THE AUTHORS. THE REVISTA ELECTRÓNICA DE LEEME AND UNIVERSITAT DE VALĖNCIA ARE NOT LIABLE FOR ANY LEGAL ACTIONS THAT MAY ARISE INVOLVING THE ARTICLE'S CONTENT. REVISTA ELECTRÓNICA DE LEEME -LISTA ELECTRÓNICA EUROPEA DE MÚSICA EN LA EDUCACIÓN-HTTP://OJS.UV.ES/INDEX.PHP/LEEME/INDEX ISSN: 1575-9563 EDITORES: UNIVERSIDAD DE VALENCIA Y JESÚS TEJADA GIMÉNEZ. VISIBILIDAD DE ESTA REVISTA: SCOPUS, EMERGING SOURCES CITATION INDEX (CLARIVATE), EBSCO, CINDOC (CSIC), CITEFACTOR, COPAC, DIALNET, DICE (CSIC), DOAJ, E-REVISTAS (CSIC), EBSCO PREMIER, ERIH+, GALE CENGAGE LEARNING, IN-RECS, IRESIE, LATINDEX, MIAR, OCLC WORLDCAT, RESH, REDIB, RILM CORE JOURNALS, SUDOC, ULRICHS, ESTA REVISTA
INSTITUCIONAL DE REDIRIS-CONSEJO SUPERIOR DE INVESTIGACIONES CIENTIFICAS Y ES DE ACCESO LIBRE. CREATIVE COMMONS LICENSE 4.O BY 
Por otra parte, respecto a los materiales enfocados a la práctica instrumental, los libros de texto no ofrecen recursos que estén pensados didácticamente para el montaje de una instrumentación. Es bien conocida la situación en los libros de texto del tipo: "Toca esta conocida melodía con tus compañeros ayudándote del playback de tu CD”. Un CD puede ser útil cuando se dominan las dificultades de una pieza instrumental, pero no ayuda a prepararla.

Las razones anteriores tienen el suficiente calado como para que el docente se plantee qué tipo de recursos serían de utilidad para que estudiantado pueda afrontar con ciertas garantías la interpretación de una pieza instrumental. Los recursos audiovisuales podrían ser un gran aliado para ello. El diseño de un recurso audiovisual pensado para el trabajo autónomo del discente, con fácil disponibilidad y accesibilidad podría evitar otro problema añadido como que este sólo pueda practicar con el instrumento con la presencia obligatoria del docente; en casa, el estudiante probablemente haya olvidado todos aquellos aspectos que se le han explicado horas o días antes. Por ello, el vídeo podría actuar a modo de 'profesor de música en casa', para ser consultado en cualquier momento y lugar y adaptándose al ritmo de aprendizaje del educando.

\subsection{Contexto y participantes}

Esta experiencia educativa se ha realizado en un instituto público de la Comunidad Autónoma de la Región de Murcia, con estudiantes de música de $4^{\circ}$ de ESO. La música en este curso era optativa y con una carga horaria semanal de 3 horas, siendo habitual que todos los años se constituyese un único curso de música en este nivel. La muestra $(n=24)$ estaba distribuida en 11 chicas y 13 chicos. La media de edad es $M=15.27$ ( $S D=.30$ ). La elección de este grupo para esta experiencia se justificó, en primer lugar, por razones de accesibilidad, siendo el docente que suscribe este trabajo el que ha impartido clases a estos estudiantes durante el curso académico 2017-2018. En segundo lugar, el libro de texto de estos alumnos contiene piezas de técnica instrumental, tanto para flauta como para láminas, lo que supone una buena oportunidad para trabajar con ellos repertorio -sobre todo en láminas- de cierta exigencia técnica.

\subsection{Diseño del estudio y herramientas para la recogida de datos}

El enfoque metodológico adoptado en este trabajo ha sido la investigación-acción (concretamente en una primera fase o espiral de ciclos). Para Kemmis (1988) la investigaciónacción debe entenderse como una investigación sobre la práctica, realizada por y para los prácticos, en este caso por el profesorado. Lomax (1995) la contempla como "una intervención en la práctica para generar una mejora" (p. 49). Siguiendo el modelo de McNiff y Whitehead (2002) se ha contemplado la investigación-acción siguiendo la siguiente secuencia: sentir o experimentar un problema, imaginar la solución del problema, poner en práctica la solución imaginada y evaluar los resultados de las acciones emprendidas.

Como herramienta de recogida de datos se ha utilizado el cuestionario. El cuestionario Percepción del alumnado sobre los audiovisuales en una metodología de clase invertida fue creado ad hoc para conocer la percepción del alumnado sobre la metodología FC, así como sobre

@JOSÉ PALAZÓN-HERRERA. THE CONTENT OF THIS ARTICLE IS THE SOLE RESPONSIBILITY OF THE AUTHORS. THE REVISTA ELECTRÓNICA DE LEEME AND UNIVERSITAT DE VALĖNCIA ARE NOT LIABLE FOR ANY LEGAL ACTIONS THAT MAY ARISE INVOLVING THE ARTICLE'S CONTENT. REVISTA ELECTRÓNICA DE LEEME -LISTA ELECTRÓNICA EUROPEA DE MÚSICA EN LA EDUCACIÓN-HTTP://OJS.UV.ES/INDEX.PHP/LEEME/INDEX ISSN: 1575-9563 EDITORES: UNIVERSIDAD DE VALENCIA Y JESÚS TEJADA GIMÉNEZ. VISIBILIDAD DE ESTA REVISTA: GALE CENGAGE LEARNING, IN-RECS, IRESIE, LATINDEX, MIAR, OCLC WORLDCAT, RESH, REDIB, RILM CORE JOURNALS, SUDOC, ULRICHS, ESTA REVISTA ESTA PUBLICADA CON EL APOYO INSTITUCIONAL DE REDIRIS-CONSEJO SUPERIOR DE INVESTIGACIONES CIENTIFICAS Y ES DE ACCESO LIBRE. CREATIVE COMMONS LICENSE 4.O BY 
el uso de vídeos en dicha metodología. El cuestionario propone como inicio una pregunta dicotómica y dos preguntas abiertas ${ }^{2}$. A continuación, se plantean trece preguntas basadas en una escala de Likert de 5 puntuaciones que van desde Muy en desacuerdo hasta Muy de acuerdo. Las variables contempladas son la 'metodología' (utilidad de las explicaciones del profesor en casa, clase invertida frente a la tradicional, adaptación al ritmo de aprendizaje, incremento del interés de estudio y mayor preparación en los ensayos) y el 'uso de vídeos como material de estudio' (claridad expositiva de los vídeos, eficacia frente a otros recursos, motivación en el visionado, duración y calidad de imagen, atención más personalizada y mayor independencia).

\subsection{Materiales}

Las herramientas tecnológicas utilizadas han sido:

- Vegas Video Pro: los vídeos del estudio de grabación han sido editados en pequeñas 'píldoras visuales' para un trabajo del alumnado más segmentado. Para evitar el uso de dos recursos diferentes, los vídeos se han complementado con partituras (Figura 1).

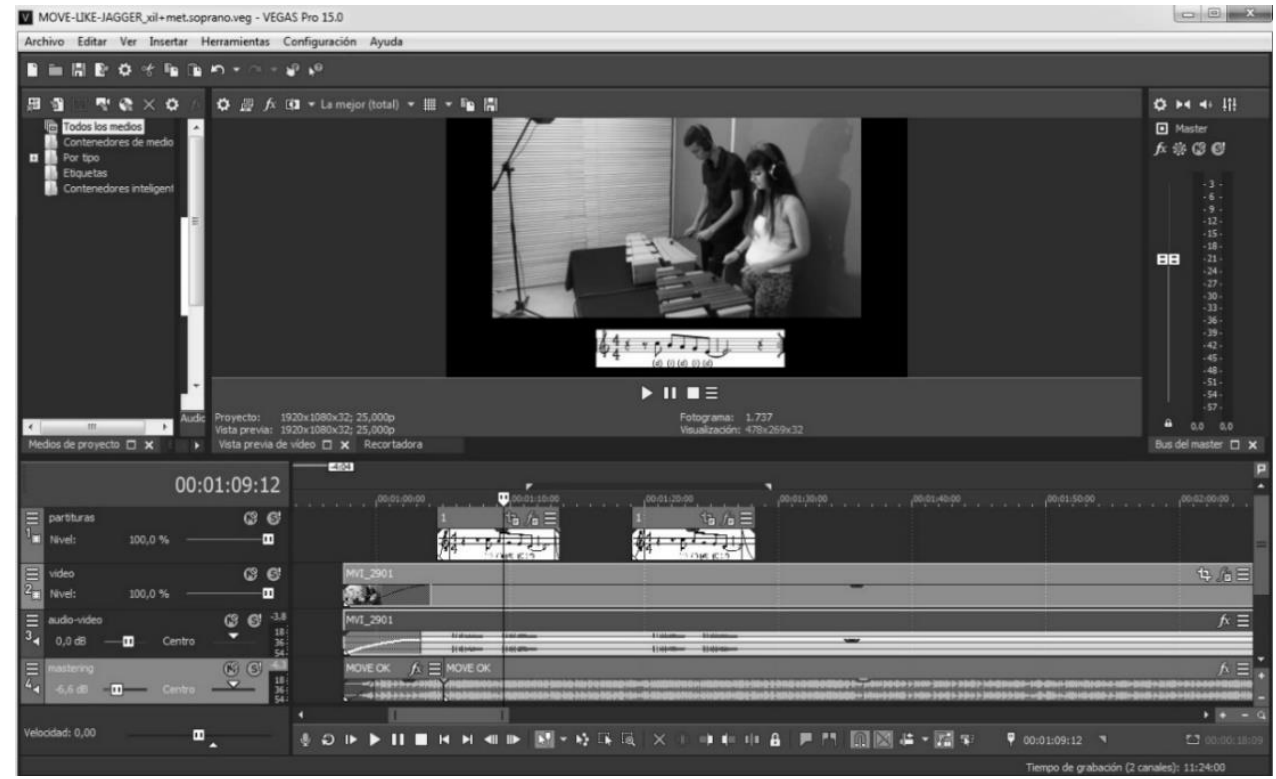

Figura 1. Pistas de audio y vídeo editadas para el trabajo instrumental en casa

- YouTube: se ha utilizado como repositorio para alojar todos los vídeos utilizados en este proyecto, creándose listas de reproducción para una mejor accesibilidad para el

\footnotetext{
${ }^{2}$ Las tres preguntas iniciales planteadas en el formulario fueron:

1. ¿Has visto vídeos en casa que contengan las explicaciones del profesor de una clase normal y has tenido que trabajarlos para preparar la siguiente clase? (respuesta SÍ/NO).

2. ¿Qué tipo de vídeos has visto en otras asignaturas como apoyo o refuerzo a las mismas? (pregunta abierta).

3. ¿qué dificultadas encuentras cuando el profesor explicar por primera vez cómo montar un tema en las láminas o en la flauta? (pregunta abierta).
} 
alumnado. El alojamiento de los vídeos en esta plataforma permitió, posteriormente, su uso en Edpuzzle.

- Edpuzzle: es una herramienta online que permite el alojamiento -y edición básica- de vídeos provenientes de canales como YouTube, Khan Academy, National Geographic, TED Talks e incluso de otros usuarios de Edpuzzle. Uno de los puntos fuertes de esta herramienta es que permite exportar los resultados de cada vídeo visto por el alumnado: porcentaje de vídeo visto, respuestas correctas e incorrectas, tiempo invertido en la visualización y número de veces que ha visto un fragmento de vídeo que contiene alguna cuestión a resolver.

- Códigos QR: se han utilizado insertados en las partituras musicales del estudiantado. Estos códigos remiten a vídeos con interpretaciones o consejos instrumentales a cargo del profesor, reforzando así los vídeos de las interpretaciones del alumnado ${ }^{3}$.

- Vídeos grabados en estudio de grabación (VEG): estos vídeos se han grabado, con anterioridad a este proyecto, por estudiantes de Secundaria y un grupo de músicos de apoyo. Además de ofrecer la interpretación grupal o de conjunto de un tema musical, también se cuenta con la grabación de cada estudiante por separado, siendo un material de una gran ayuda para el alumnado, el cual puede elegir entre varios instrumentos para su estudio. Estos vídeos (los de las interpretaciones de conjunto) son como 'partituras audiovisuales'4 pues los instrumentistas van apareciendo o desapareciendo en pantalla según el arreglo en partitura que se ha realizado.

- Vídeos grabados por el profesor (VP): en estos vídeos el profesorado muestra cómo interpretar cualquier fragmento de los vídeos grabados en el estudio de grabación ${ }^{5}$.

- Vídeos originales (VO): vídeos con la interpretación de los artistas originales.

\subsection{Procedimiento}

Respecto al desarrollo de la experiencia, el alumnado fue informado de que iban a trabajar la práctica instrumental de una manera diferente a como lo venían haciendo. Esta nueva manera de trabajar implicaría no sólo el uso de ciertas tecnologías de apoyo al trabajo instrumental sino, también, un cambio en la metodología empleada hasta el momento. Así, para el montaje de una pieza instrumental se siguió siempre la secuencia que se muestra a continuación:

1. [Clase 1] El profesor proyectó un vídeo (VO) -como elemento motivador- con la interpretación original de uno de los temas musicales que el alumnado iba a tocar con los instrumentos del aula (instrumentos de láminas y flauta), en este caso Move Like Jagger, versión de Maroon 5 con Christina Aguilera.

2. [Clase 1-continuación-] El profesor proyectó la interpretación instrumental del tema elegido a cargo de un grupo de estudiantes de Secundaria, para que vieran el resultado final del tema original en su versión para instrumentos del aula (VEG) (ver Figura 2). Esta proyección

\footnotetext{
3 Puede verse un ejemplo (extracto) de una de las partituras con códigos QR en: https://bit.ly/2M7rVKB

4 Puede verse el vídeo grabado en estudio por estudiantes de Secundaria en: https://bit.ly/2I4ggyJ

5 Puede verse un ejemplo de vídeo grabado por el docente en: https://bit.ly/2oQant4
} 
pretendía ser otro elemento motivador para ellos, que podían ver a alumnado de Secundaria tocando temas de música moderna con instrumentos del aula, con un sonido profesional y con una puesta visual en escena muy sugerente, además de didáctica. Estos vídeos sirvieron para que cada estudiante, en función de su motivación hacia el aprendizaje de un determinado instrumento o de la dificultad que pudiera suponer el aprendizaje de determinadas partes instrumentales, eligiera qué instrumento quería tocar en la canción elegida.

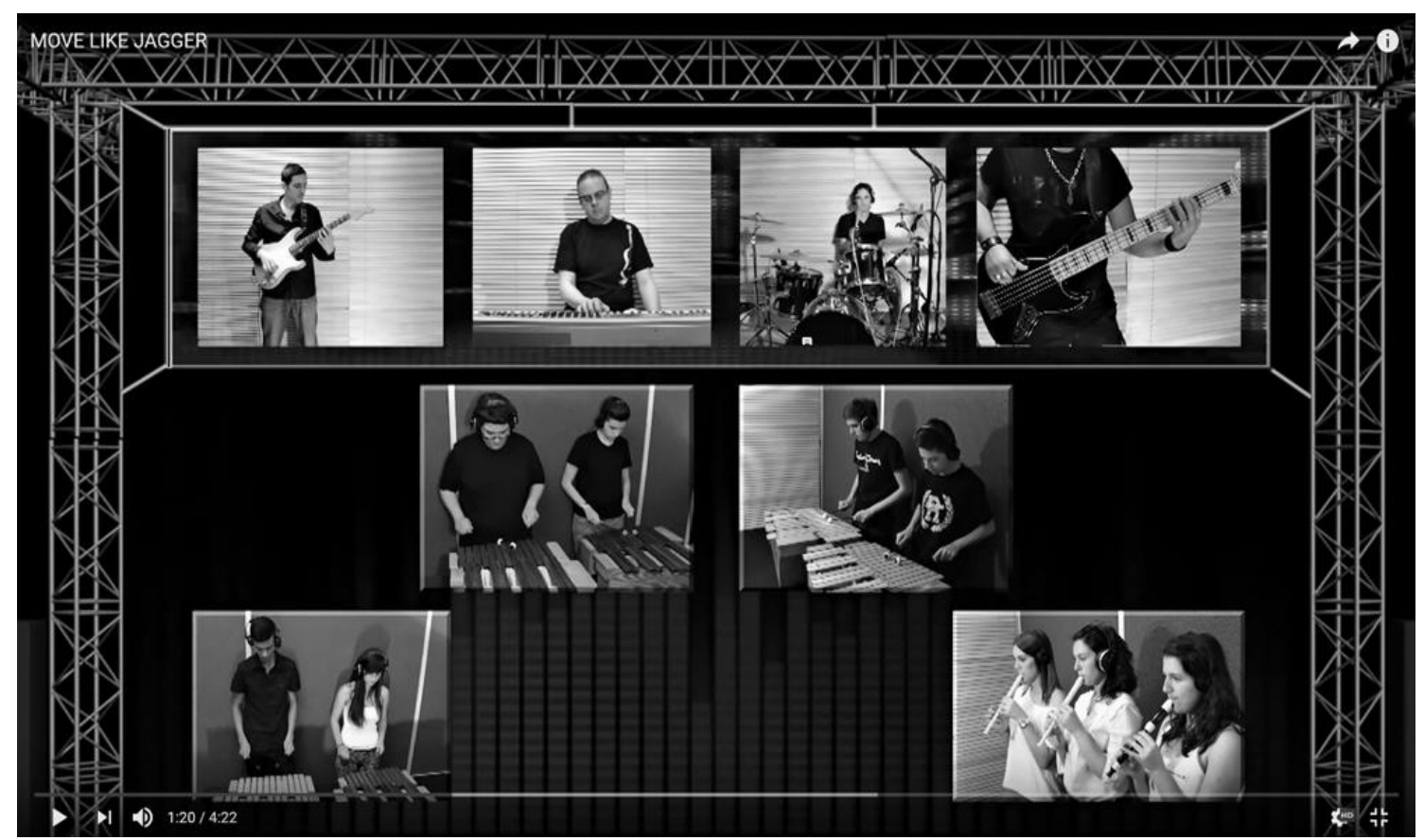

Figura 2. Captura de vídeo de la interpretación del tema Move Like Jagger, de Maroon 5, a cargo de alumnos de Secundaria

El profesor (ver punto 4) comentó al alumnado que estos vídeos (VEG) iban a ser alojados en Edpuzzle para que pudieran estudiarlos en casa. Igualmente, les recordaba su compromiso hacia el visionado obligatorio -al menos una vez- y estudio de estos vídeos en casa, ya que debían realizar las actividades que aparecían en los mismos, y esto quedaba reflejado en la plataforma Edpuzzle. La idea era garantizar que el alumnado llegase a la siguiente clase presencial con cierta preparación, de lo contrario no se cumpliría una de las premisas básicas del enfoque FC. Por ello, se informó al estudiantado que este visionado de los vídeos en casa se utilizaría como un elemento más de evaluación de su trabajo.

3. El docente subió los vídeos de la canción a trabajar (VEG) primeramente a YouTube para que, posteriormente, pudieran ser insertados en Edpuzzle y, por tanto, disponibles para el alumnado, para que pudieran verlos en cualquier momento y lugar y las veces que necesitara.

4. [En casa: 1] El estudiantado debía visionar en la plataforma Edpuzzle el VEG que hubiese elegido y contestar a las preguntas que en el mismo se formulasen. En el caso de los instrumentos de láminas -que son los instrumentos más problemáticos, pues son los que menos suelen utilizar los alumnos-, los discentes debían contestar en el vídeo sobre qué combinación de manos se había utilizado para la interpretación de un determinado pasaje instrumental (Figura 3).

@JOSÉ PALAZÓN-HERRERA. THE CONTENT OF THIS ARTICLE IS THE SOLE RESPONSIBILITY OF THE AUTHORS. THE REVISTA ELECTRÓNICA DE LEEME AND UNIVERSITAT DE VALĖNCIA ARE NOT LIABLE FOR ANY LEGAL ACTIONS THAT MAY ARISE INVOLVING THE ARTICLE'S CONTENT. REVISTA ELECTRÓNICA DE LEEME - LISTA ELECTRÓNICA EUROPEA DE MÚSICA EN LA EDUCACIÓN-HTTP://OJS.UV.ES/INDEX.PHP/LEEME/INDEX ISSN: 1575-9563 EDITORES: UNIVERSIDAD DE VALENCIA Y JESÚS TEJADA GIMÉNEZ. VISIBILIDAD DE ESTA REVISTA: SCOPUS, EMERGING SOURCES CITATION INDEX (CLARIVATE), EBSCO, CINDOC (CSIC), CITEFACTOR, COPAC, DIALNET, DICE (CSIC), DOAJ, E-REVISTAS (CSIC), EBSCO PREMIER, ERIH+, GALE CENGAGE LEARNING, IN-RECS, IRESIE, LATINDEX, MIAR, OCLC WORLDCAT, RESH, REDIB, RILM CORE JOURNALS, SUDOC, ULRICHS, ESTA REVISTA ESTÁ PUBLICADA CON EL APOYO INSTITUCIONAL DE REDIRIS-CONSEJO SUPERIOR DE INVESTIGACIONES CIENTIFICAS Y ES DE ACCESO LIBRE. CREATIVE COMMONS LICENSE 4.0 BY 
Esto les obligaba a visionar el vídeo varias veces familiarizándose con diferentes aspectos de la canción y repasando la disposición de manos que debían utilizar para tocar un determinado fragmento.

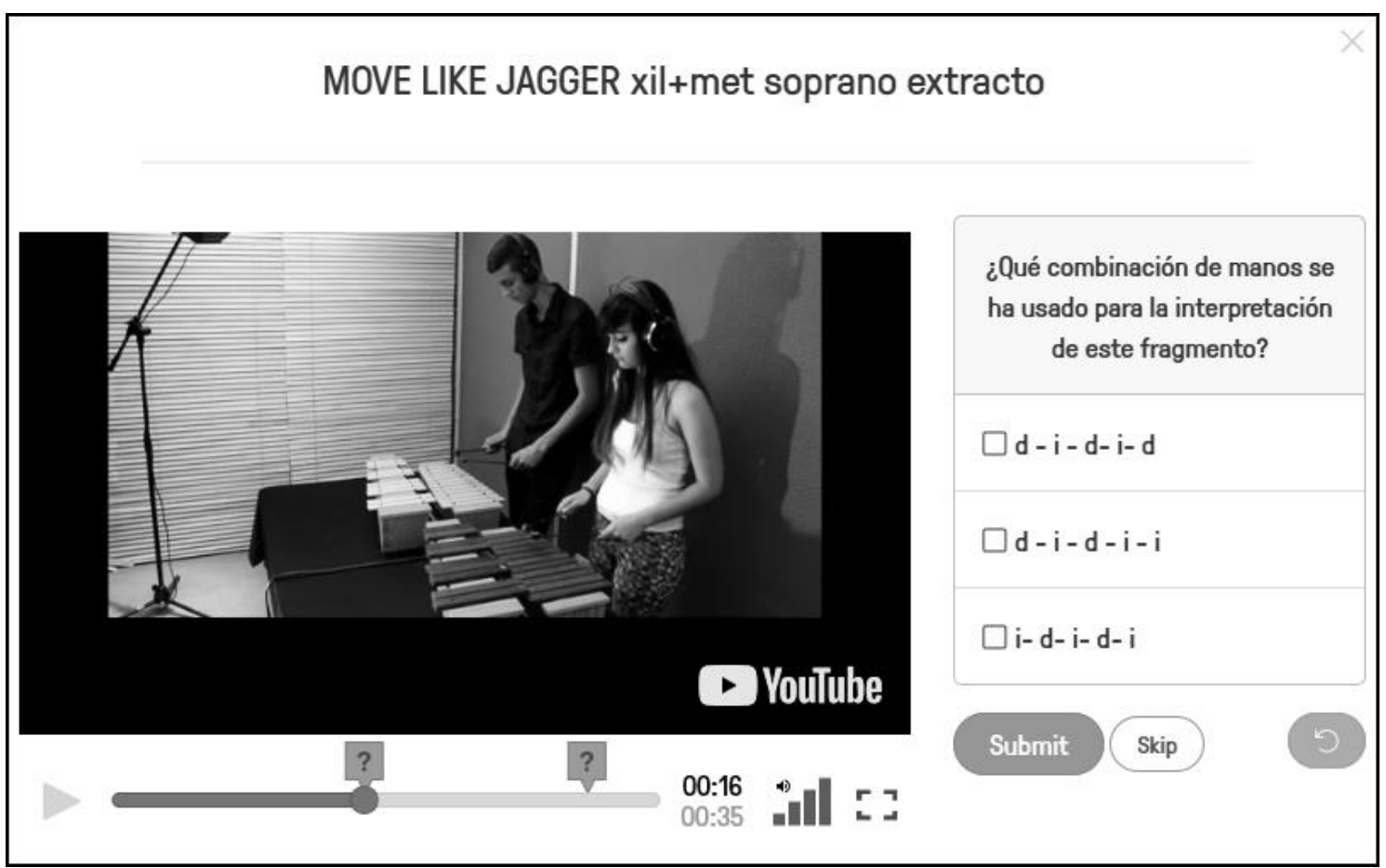

Figura 3. Captura de vídeo (VEG) en Edpuzzle, donde el alumnado debe estudiar su parte instrumental y contestar las cuestiones que se le van planteando durante el visionado del vídeo

Otra pregunta que aparecía en todos los vídeos - pregunta abierta- era qué posible combinación de manos utilizaría él para dar respuesta a ese mismo fragmento musical, lo que obligaba al alumno a plantearse en casa posibles alternativas a la propuesta del profesor, propiciando que el alumno reflexionara sobre cómo solucionar un problema interpretativo.

5. [Clase 2] El profesor volvía a proyectar el vídeo (VEG) con la interpretación grupal del tema musical a cargo de estudiantes de Secundaria, para que se familiarizasen más con la estructura del tema, sus motivos melódicos, etc., a la vez que iban recordando lo trabajado en casa. A continuación, el alumnado, bien en sus mesas de trabajo ${ }^{6}$ o bien colocados en los instrumentos del aula, practicaron con ellos utilizando partituras enriquecidas con códigos QR. El procedimiento fue el siguiente: el estudiantado, con sus dispositivos móviles (usando auriculares para no molestarse acústicamente unos a otros) escaneaban estos códigos $\mathrm{QR}^{7}$, los cuales remitían a vídeos grabados por su profesor mostrando cómo interpretar un determinado fragmento, resolviendo así cualquier duda del alumnado. En ese momento, el profesor disponía de mucho

\footnotetext{
${ }^{6}$ Todo el alumnado disponía desde principios de curso de folios tamaño DIN-A3 con la imagen de un instrumento de láminas para poder practicar mientras no había disponibilidad de instrumento en el aula.

7 El alumnado instaló la app i-nigma en sus smartphones para este proyecto.
}

@JOSÉ PALAZÓN-HERRERA. THE CONTENT OF THIS ARTICLE IS THE SOLE RESPONSIBILITY OF THE AUTHORS. THE REVISTA ELECTRÓNICA DE LEEME AND UNIVERSITAT DE VALĖNCIA ARE NOT LIABLE FOR ANY LEGAL ACTIONS THAT MAY ARISE INVOLVING THE ARTICLE'S CONTENT. REVISTA ELECTRÓNICA DE LEEME -LISTA ELECTRÓNICA EUROPEA DE MÚSICA EN LA EDUCACIÓN-HTTP://OJS.UV.ES/INDEX.PHP/LEEME/INDEX ISSN: 1575-9563 EDITORES: UNIVERSIDAD DE VALENCIA Y JESÚS TEJADA GIMÉNEZ. VISIBILIDAD DE ESTA REVISTA: GALE CENGAGE LEARNING, INERECS, IRESIE, LATINDEX, MIAR, OCLC WORLDCAT, RESH, REDIB, RILM CORE JOURNALS, SUDOC, ULRICHS, ESTA REVISTA ESTÁ PUBLICADA CON EL APOYO INSTITUCIONAL DE REDIRIS-CONSEJO SUPERIOR DE INVESTIGACIONES CIENTIFICAS Y ES DE ACCESO LIBRE. CREATIVE COMMONS LICENSE 4.O BY 
más tiempo para corregir las dudas puntuales que pudieran surgir, pues los discentes contaban con 'un profesor particular' en el aula (los vídeos grabados con las explicaciones del profesor), pudiendo el profesor aportar feedback de manera mucho más eficiente.

6. [En casa 2] El alumnado debía repasar todo lo trabajado anteriormente para prepararse para los ensayos a nivel grupal en el aula. Para ello, repasaban de nuevo el vídeo VEG de la interpretación grupal y la partitura individual según el vídeo VEG. El alumnado ya debía disponer de una perspectiva más madura tanto del tema musical en su conjunto como de la parte interpretativa que, a nivel individual, debía controlar, pues había trabajado varias veces su parte y había escuchado el tema musical repetidamente.

7. [Clase 3] El profesor -si el estudiantado dominaba razonablemente el contenido a interpretar- comenzaba con los primeros ensayos grupales, si no, debía seguir alguna sesión más con el trabajo individual. Si el alumnado estaba preparado para comenzar con ensayos parciales o grupales, una forma de emprender este recorrido fue: trabajar al unísono aquellos instrumentos que tenían una partitura con contenido similar o idéntico (por ejemplo, en el tema de Maroon 5 el xilófono soprano y el metalófono soprano tocan lo mismo en la partitura). Y a partir de ese momento, ir sumando instrumentos de uno en uno, trabajando siempre con un control riguroso del pulso -aconsejable siempre la interpretación en varios tempi de menor a mayor velocidadhasta poder llegar a tocar con el playback cada alumno su parte a la velocidad original.

8. [En casa: 3 y 4] Las partes instrumentales del xilófono y metalófono altos -para el tema Move Like Jagger- requirieron por su dificultad (ver Figura 4) de dos sesiones extra en casa para resolver el pasaje instrumental central; por ello, se aconsejó al alumnado que estudiara este pasaje exclusivamente con los vídeos grabados por el profesor. A continuación, podemos ver este fragmento musical, que presenta una exigencia técnica considerable para este nivel educativo ${ }^{8}$. 

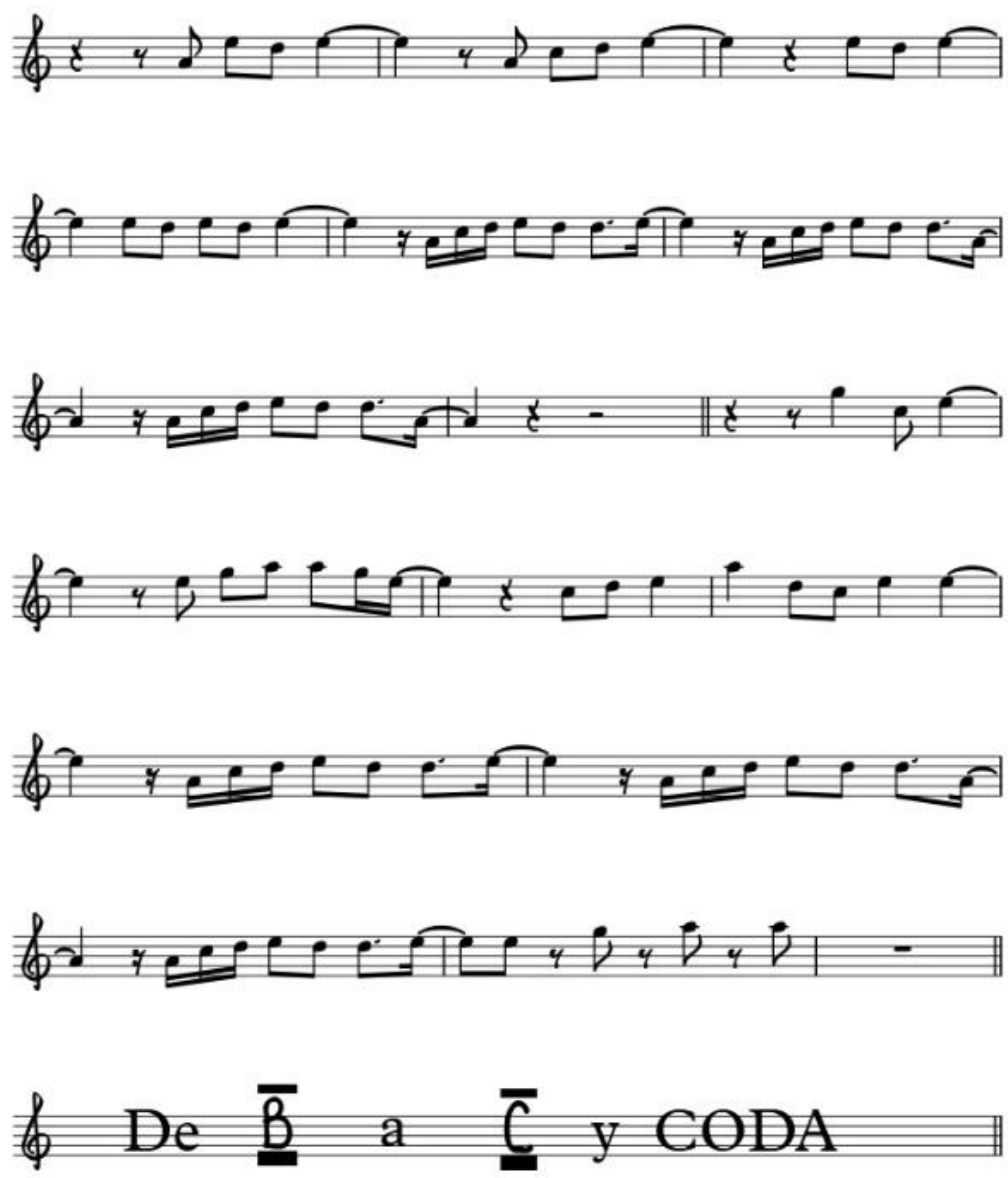

Figura 4. Fragmento central en el tema Move Lake Jagger (xilófono y metalófono altos)

9. Se volvería a repetir el proceso anterior en función de las necesidades o dificultades de los alumnos, avanzando hacia la interpretación grupal final con el playback.

\section{Resultados}

A continuación, se presentan los resultados en función de los objetivos propuestos en este trabajo. En primer lugar, y con respecto a las dos primeras preguntas abiertas formuladas al alumnado antes de comenzar a implantar la metodología FC para el estudio instrumental, éstos respondieron que nunca habían utilizado vídeos con las explicaciones de sus docentes como material de apoyo. El $100 \%$ del estudiantado afirmó que el vídeo no era un recurso que el profesorado mandara como 'deberes' para casa, pero sí que los proyectaban ocasionalmente en clase, tratándose la mayor parte de las ocasiones de fragmentos de películas -o películas completas- o algún vídeo de YouTube como introducción o refuerzo de algún tema que estaban trabajando. Al finalizar la visualización del vídeo o película, el docente les formulaba alguna

@JOSÉ PALAZÓN-HERRERA. THE CONTENT OF THIS ARTICLE IS THE SOLE RESPONSIBILITY OF THE AUTHORS. THE REVISTA ELECTRÓNICA DE LEEME AND UNIVERSITAT DE VALĖNCIA ARE NOT LIABLE FOR ANY LEGAL ACTIONS THAT MAY ARISE INVOLVING THE ARTICLE'S CONTENT. REVISTA ELECTRÓNICA DE LEEME -LISTA ELECTRÓNICA EUROPEA DE MÚSICA EN LA EDUCACIÓN-HTTP://OJS.UV.ES/INDEX.PHP/LEEME/INDEX ISSN: 1575-9563 EDITORES: UNIVERSIDAD DE VALENCIA Y JESÚS TEJADA GIMÉNEZ. VISIBILIDAD DE ESTA REVISTA: SCOPUS, EMERGING SOURCES CITATION INDEX (CLARIVATE), EBSCO, CINDOC (CSIC), CITEFACTOR, COPAC, DIALNET, DICE (CSIC), DOAJ, E-REVISTAS (CSA), LEICADA CON EL APOYO INSTITUCIONAL DE REDIRIS-CONSEJO SUPERIOR DE INVESTIGACIONES CIENTIFICAS Y ES DE ACCESO LIBRE. CREATIVE COMMONS LICENSE 4.0 BY 
pregunta sobre el contenido visto. En ningún caso, el vídeo contenía las explicaciones del profesorado sobre un determinado tema ni servía para preparar la siguiente clase.

Respecto a la tercera pregunta abierta (dificultades que el alumnado se encontraba a la hora de enfrentarse por primera vez al montaje de una pieza instrumental), a continuación, se resumen las contestaciones que dio el estudiantado:

- Es difícil seguir la explicación que da el docente, aun cuando la explicación o demostración la hace en un instrumento o en la pizarra, pues somos muchos y es muy fácil despistarse y perder la concentración u olvidar cómo hay que hacerlo.

- La explicación del docente sólo la vemos una vez, por lo que cuando estamos en casa hemos olvidado muchos de los detalles que nos ha explicado en clase.

- Cuesta mucho avanzar porque de una clase a otra se nos olvidan tantos detalles que el docente debe volver a explicar de nuevo muchas cosas que ya se habían explicado, por lo que se pierde mucho tiempo.

En lo que se refiere al cuestionario Percepción del alumnado sobre los audiovisuales en una metodología de clase invertida, se analizan dos dimensiones: la metodología FC y el vídeo como recurso en dicha metodología. Para medir cada dimensión se ha pedido a los 24 estudiantes participantes que valoren estas dimensiones según una escala Likert de cinco puntuaciones que van desde Muy en desacuerdo (1) a Muy de acuerdo (5).

Para la dimensión metodología FC se han recogido los aspectos: 'Utilidad para el alumno de las explicaciones en casa del profesor', 'Adaptación de la metodología a su ritmo de aprendizaje', 'Incremento del interés del alumno hacia la práctica instrumental' y 'Mejor preparación a la hora de enfrentarse a la siguiente clase presencial'. En el siguiente gráfico (Figura 5), se muestra la media para cada indicador:

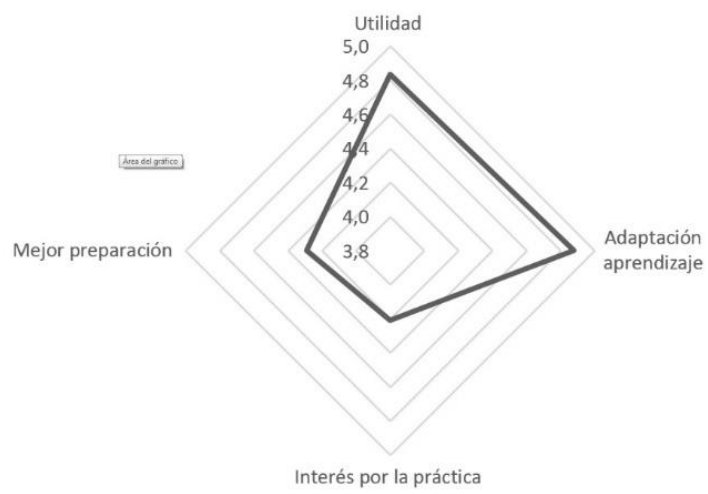

Figura 5. Medias de los diferentes indicadores sobre la metodología FC

El gráfico de la Figura 5 permite comparar cuál de los cuatro aspectos obtiene una mejor valoración, observándose que la 'Adaptación al ritmo de aprendizaje' $(M=4.88)$ y la 'Utilidad en casa de las explicaciones del profesor’ $(M=4.83)$ obtienen la puntuación más alta por parte de la muestra estudiada, estando sobre una media de 4.2 los indicadores 'Mayor preparación' e 'Interés

@JOSÉ PALAZÓN-HERRERA. THE CONTENT OF THIS ARTICLE IS THE SOLE RESPONSIBILITY OF THE AUTHORS. THE REVISTA ELECTRÓNICA DE LEEME AND UNIVERSITAT DE VALĖNCIA ARE NOT LIABLE FOR ANY LEGAL ACTIONS THAT MAY ARISE INVOLVING THE ARTICLE'S CONTENT. REVISTA ELECTRÓNICA DE LEEME -LISTA ELECTRÓNICA EUROPEA DE MÚSICA EN LA EDUCACIÓN-HTTP://OJS.UV.ES/INDEX.PHP/LEEME/INDEX ISSN: 1575-9563 EDITORES: UNIVERSIDAD DE VALENCIA Y JESÚS TEJADA GIMÉNEZ. VISIBILIDAD DE ESTA REVISTA: SCOPUS, EMERGING SOURCES CITATION INDEX (CLARIVATE), EBSCO, CINDOC (CSIC), CITEFACTOR, COPAC, DIALNET, DICE (CSIC), DOAJ, E-REVISTAS (CSIC), EBSCO PREMIER, ERIH+, GALE CENGAGE LEARNING, IN-RECS, IRESIE, LATINDEX, MIAR, OCLC WORLDCAT, RESH, REDIB, RILM CORE JOURNALS, SUDOC, ULRICHS, ESTA REVISTA
INSTITUCIONAL DE REDIRIS-CONSEJO SUPERIOR DE INVESTIGACIONES CIENTIFICAS Y ES DE ACCESO LIBRE. CREATIVE COMMONS LICENSE 4.0 BY 
por la práctica instrumental'. En cualquier caso, estos cuatro aspectos obtienen una alta valoración alta, a partir de 4 (De acuerdo).

Igualmente, se pidió al alumnado que valorase la metodología FC (Figura 6) comparándola con la metodología tradicional expositiva. El alumnado valoró con un porcentaje cercano al 90\% las opciones De acuerdo y Muy de acuerdo en su preferencia del enfoque FC sobre el modelo expositivo tradicional para la práctica instrumental.

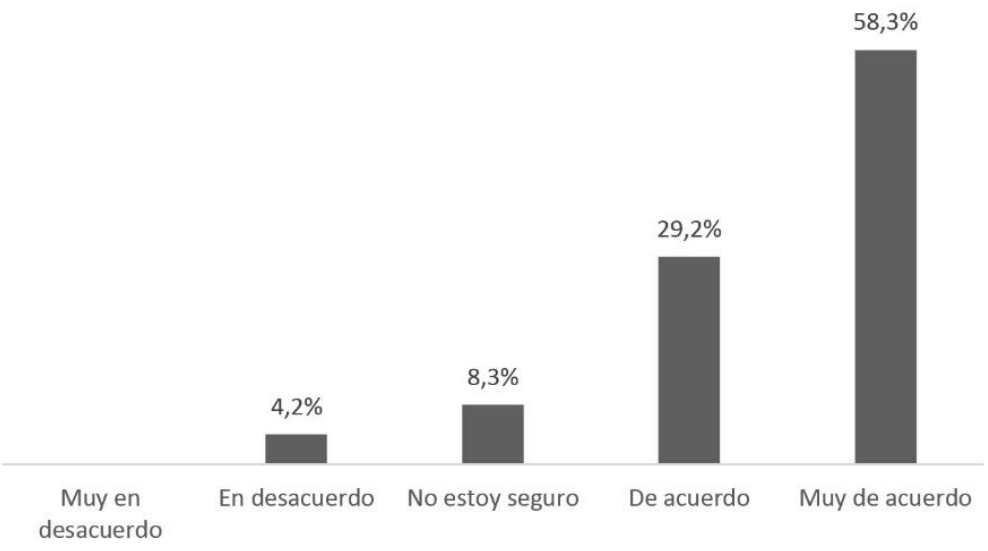

Figura 6. Preferencia del modelo FC frente a la clase tradicional expositiva.

Para la medición del vídeo como recurso de apoyo a la práctica instrumental, se han recogido los siguientes aspectos: 'Es similar en contenido a una clase', 'Aumentan la confianza para el trabajo en la siguiente clase', 'Su uso es motivador', 'Permite al profesor tener más tiempo para atender a sus alumnos', 'Tienen buena calidad', 'Tienen una duración adecuada', 'Usar dispositivos móviles con estos vídeos me hace más independiente del profesor'. A continuación (Figura 7) se muestra un gráfico radial de medias sobre la valoración de los alumnos en cada uno de los aspectos mencionados.

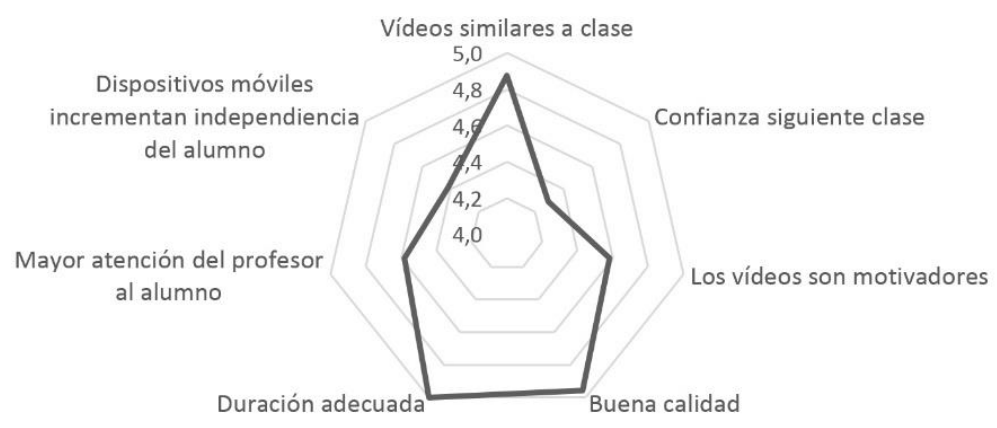

Figura 7. Valoración del alumnado sobre el vídeo como recurso en la metodología FC

Se puede observar en la Figura 7 que los siete indicadores obtuvieron una media superior a 4.2, siendo la duración y la buena calidad de los vídeos los que obtuvieron la máxima puntuación

@JOSÉ PALAZÓN-HERRERA. THE CONTENT OF THIS ARTICLE IS THE SOLE RESPONSIBILITY OF THE AUTHORS. THE REVISTA ELECTRÓNICA DE LEEME AND UNIVERSITAT DE VALĖNCIA ARE NOT LIABLE FOR ANY LEGAL ACTIONS THAT MAY ARISE INVOLVING THE ARTICLE'S CONTENT. REVISTA ELECTRÓNICA DE LEEME -LISTA ELECTRÓNICA EUROPEA DE MÚSICA EN LA EDUCACIÓN-HTTP://OJS.UV.ES/INDEX.PHP/LEEME/INDEX ISSN: 1575-9563 EDITORES: UNIVERSIDAD DE VALENCIA Y JESÚS TEJADA GIMÉNEZ. VISIBILIDAD DE ESTA REVISTA: SCOPUS, EMERGING SOURCES CITATION INDEX (CLARIVATE), EBSCO, CINDOC (CSIC), CITEFACTOR, COPAC, DIALNET, DICE (CSIC), DOAJ, E-REVISTAS (CSIC), EBSCO PREMIER, ERIH+, INSTITUCIONAL DE REDIRIS-CONSEJO SUPERIOR DE INVESTIGACIONES CIENTIFICAS Y ES DE ACCESO LIBRE. CREATIVE COMMONS LICENSE 4.O BY 
$(M=5)$, seguido de 'Los vídeos son similares a las clases presenciales' $(M=4.9)$, mientras que el ítem con la valoración más baja $(M=4.3)$ fue 'Los vídeos contribuyen a que vaya con más confianza a la siguiente clase'.

Por último, se pidió al alumnado que valorase si 'Los vídeos son más adecuados que otros recursos -apuntes, libro de texto, presentaciones PowerPoint o similares- para la práctica instrumental', dándose un $100 \%$ en la puntuación Muy de acuerdo por parte de éste.

\section{Conclusiones}

La búsqueda de soluciones hacia el reto de la interpretación instrumental en un nivel educativo como la Educación Secundaria obliga a replantearnos como docentes cuáles son las necesidades y problemas de nuestro alumnado y qué recursos y metodologías se adaptan mejor a esta situación.

El estudiantado participante en este proyecto venía expresando a lo largo del curso la dificultad que les suponía intentar retener las explicaciones del docente y llevarlas a la práctica a la hora de montar una pieza instrumental, lo que dificultaba sobremanera un desarrollo razonable de las siguientes clases presenciales. Ello obligaba al docente que esto suscribe a repetir en la siguiente o siguientes clases muchos aspectos que ya se habían explicado, lo que no sólo suponía un importante esfuerzo a nivel personal, sino que alargaba indebidamente el montaje de una pieza, limitando además enormemente la experimentación del alumnado sobre un repertorio variado. Por ello, el diseño de vídeos pensados específicamente para este fin ha demostrado ser una ayuda muy valiosa para el alumnado, el cual ha valorado este recurso de manera muy positiva, no sólo por sus características técnicas (como que sean breves, accesibles y con las menores deficiencias posibles, en consonancia con trabajos como los de Long, Logan y Waugh, 2016), sino también por el hecho de que algunos de los vídeos que se utilizaron eran una 'simulación' de lo que el profesor explicaba en clase, facilitando el trabajo individual cuando se utilizaba como material pre-clase (en sintonía con trabajos como el de Imran, 2013), despejando así la mayor parte de las dudas que solían suscitarse en el trabajo instrumental, siendo éste uno de sus puntos fuertes. De esta manera, el alumnado tuvo a su disposición el contenido que necesitaba y cuando lo necesitaba para estudiarlo las veces que fuera necesario, adaptándose perfectamente tanto en clase como en casa a su ritmo de aprendizaje. Esto supuso un primer 'volteamiento' o inversión de la clase, pues ya no hay un docente que explica para 30 alumnos sino ' 30 docentes virtuales, uno para cada alumno'.

El diseño de estos recursos puede suponer en muchas ocasiones un auténtico reto para el profesorado. En primer lugar, y aunque pensemos que en YouTube -por citar uno de los repositorios de vídeos por excelencia- se encuentra todo, lo cierto es que, si queremos montar piezas instrumentales muy concretas, seguro que no podremos encontrar el vídeo que se adapte a lo que queremos enseñar a nuestro estudiantado, por lo que nos veremos en la necesidad de crearlo nosotros si queremos un resultado concreto. Esto exige, lógicamente, que el profesorado dé un

@JOSÉ PALAZÓN-HERRERA. THE CONTENT OF THIS ARTICLE IS THE SOLE RESPONSIBILITY OF THE AUTHORS. THE REVISTA ELECTRÓNICA DE LEEME AND UNIVERSITAT DE VALĖNCIA ARE NOT LIABLE FOR ANY LEGAL ACTIONS THAT MAY ARISE INVOLVING THE ARTICLE'S CONTENT. REVISTA ELECTRÓNICA DE LEEME -LISTA ELECTRÓNICA EUROPEA DE MÚSICA EN LA EDUCACIÓN-HTTP://OJS.UV.ES/INDEX.PHP/LEEME/INDEX ISSN: 1575-9563 EDITORES: UNIVERSIDAD DE VALENCIA Y JESÚS TEJADA GIMÉNEZ. VISIBILIDAD DE ESTA REVISTA:
SCOPUS, EMERGING SOURCES CITATION INDEX (CLARIVATE), EBSCO, CINDOC (CSIC), CITEFACTOR, COPAC, DIALNET, DICE (CSIC), DOAJ, E-REVISTAS (CSIC), EBSCO PREMIER, ERIH+, GALE CENGAGE LEARNING, IN-RECS, IRESIE, LATINDEX, MIAR, OCLC WORLDCAT, RESH, REDIB, RILM CORE JOURNALS, SUDOC, ULRICHS, ESTA REVISTA ESTÁ PUBLICADA CON EL APOYO INSTITUCIONAL DE REDIRIS-CONSEJO SUPERIOR DE INVESTIGACIONES CIENTIFICAS Y ES DE ACCESO LIBRE. CREATIVE COMMONS LICENSE 4.0 BY 
primer paso encaminado a grabarse personalmente en vídeo, lo cual todavía no es una actitud generalizada. En la actualidad, solemos grabar mucho al alumnado y, sin embargo, no nos grabamos a nosotros mismos en un ámbito de trabajo tan importante como la práctica instrumental, pues el alumnado no sólo necesita escuchar, sino que puede aprender también imitando un modelo y en este sentido el vídeo puede ser el mejor aliado. Por extensión, crear estos vídeos y seleccionar aquellas tecnologías que estén al servicio de un planteamiento concreto de enseñanza-aprendizaje como el que aquí se expone, exige del docente un cierto conocimiento y manejo de dichas tecnologías.

En este proyecto se han utilizado: un editor de partituras, un editor de vídeo, una aplicación para generar códigos QR, YouTube para crear listas de reproducción para los alumnos y una plataforma para alojar los vídeos de estudio, plataforma que permitió hacer un seguimiento del visionado de vídeos por parte del alumno, además de ofrecer interactividad dentro de los mismos a través de cuestionarios que debía cumplimentar, Lógicamente, esto requiere de un firme compromiso del docente por estar al día en el manejo de ciertas tecnologías, pues este enfoque metodológico se apoya en gran medida en su uso, tal y como subrayan Aidinopoulou y Sampson (2017), exigiendo por ello una inversión de tiempo que no siempre el docente está dispuesto a asumir.

Respecto a la metodología flipped classroom, el alumnado ha respondido muy positivamente a la misma. Aunque al comienzo del proyecto se albergaban dudas sobre el compromiso del estudiante hacia el estudio de los vídeos en casa con vista a un trabajo más independiente en el aula, los resultados subrayan que se han sentido muy motivados hacia el estudio de este recurso. El alumnado intuyó desde un principio que disponer de este material le permitiría practicar con el instrumento más tiempo en clase del habitual, lo que podría mejorar su destreza y adquirir una mayor confianza con el instrumento. Por extensión, esto permitió al docente atender de manera más personalizada a sus estudiantes, explicando o haciendo alguna demostración puntual en el instrumento, pero no viéndose en la necesidad de repetir todo un proceso que ya se había explicado con anterioridad, lo que podemos considerar como otro logro en relación a la metodología adoptada.

Los resultados de la encuesta han demostrado que el enfoque FC, según el alumnado, ayudó a optimizar mucho más el rendimiento tanto en casa como en clase, contribuyendo a una mejor preparación de las clases presenciales. En definitiva, esta metodología ha contribuido a convertir el aula en un auténtico 'laboratorio' de trabajo y experimentación, donde cada estudiante ha dispuesto de recursos que han favorecido su auto-aprendizaje y una mayor independencia con respecto al profesorado, lo que viene a reafirmar trabajos como los de Bergmann, Overmyer y Wilie (2013), quienes subrayan que el modelo FC propicia un escenario donde el estudiantado toma la responsabilidad de su aprendizaje asumiendo un rol protagonista mientras que el docente se convierte en un guía del proceso.

Finalmente, subrayar que el hecho de que el alumnado haya conseguido tocar grupalmente las piezas propuestas bajo esta metodología y con el uso del vídeo como principal recurso de apoyo, hace pensar y creer firmemente que el planteamiento adoptado ha sido correcto.

@JOSÉ PALAZÓN-HERRERA. THE CONTENT OF THIS ARTICLE IS THE SOLE RESPONSIBILITY OF THE AUTHORS. THE REVISTA ELECTRÓNICA DE LEEME AND UNIVERSITAT DE VALĖNCIA ARE NOT LARLER ANY LEGAL ACTIONS THAT MAY ARISE INVOLVING THE ARTICLE'S CONTENT. REVISTA ELECTRÓNICA DE LEEME —LISTA ELECTRÓNICA EUROPEA DE MÚSICA EN LA EDUCACIÓN-HTTP://OJS.UV.ES/INDEX.PHP/LEEME/INDEX ISSN: 1575-9563 EDITORES: UNIVERSIDAD DE VALENCIA Y JESÚS TEJADA GIMÉNEZ. VISIBILIDAD DE ESTA REVISTA: SCOPUS, EMERGING SOURCES CITATION INDEX (CLARIVATE), EBSCO, CINDOC (CSIC), CITEFACTOR, COPAC, DIALNET, DICE (CSIC), DOAJ, E-REVISTAS (CSIC), EBSCO PREMIER, ERIH+, GALE CENGAGE LEARNING, IN-RECS, IRESIE, LATINDEX, MIAR, OCLC WORLDCAT, RESH, REDIB, RILM CORE JOURNALS, SUDOC, ULRICHS, ESTA REVISTA ESTÁ PUBLICADA CON EL APOYO INSTITUCIONAL DE REDIRIS-CONSEJO SUPERIOR DE INVESTIGACIONES CIENTIFICAS Y ES DE ACCESO LIBRE. CREATIVE COMMONS LICENSE 4.0 BY 
Sin embargo, se hace necesario seguir investigando en esta línea para contestar interrogantes tales como si esta metodología realmente optimiza los tiempos de aprendizaje del alumnado, si mejora su rendimiento interpretativo a nivel técnico e incluso si podríamos plantearnos que todo el estudiantado -en circunstancias similares a las propuestas en este trabajo- conseguiría superar los objetivos propuestos, aunque como docentes tuviéramos que plantearnos diferentes tiempos de evaluación para estudiantes de un mismo grupo. En este proyecto, todo el alumnado 'ha tenido que llegar al objetivo propuesto al mismo tiempo', lo cual podría solucionarse con un modelo FC adaptativo, propuesta que también podrá ser tenida en cuenta en futuras experiencias.

\section{Referencias}

Aidinopoulou, V. y Sampson, D. G. (2017). An Action Research Study from Implementing the Flipped Classroom Model in Primary School History Teaching and Learning. Educational Technology \& Society, 20 (1), 237-247. Recuperado de https://espace.curtin.edu.au/

Bergmann, J., Overmyer, J. y Wilie, B. (2013). The Flipped Class: What it is and what is not. Recuperado de http://www.thedailyriff.com/

Bergmann, J. y Sams, A. (2012). Flip Your Classroom. Reach Every Student in Every Class Every Day. Arlington, USA: International Society for Technology in Education (ISTE).

Brame, C. J. (2016). Effective Educational Videos: Principles and Guidelines for Maximizing Student Learning from Video Content. CBE Life Sci Educ, 15 (4), es6. doi: 10.1187/cbe.16-030125

Calvillo, A. (2014). El modelo Flipped Learning aplicado a la materia de música en el cuarto curso de Educación Secundaria Obligatoria: una investigación-acción para la mejora de la práctica docente y del rendimiento académico del alumnado (Tesis doctoral). Segovia: Universidad de Valladolid. Recuperado de https://www.educacion.gob.es/teseo/

Chen, Y., Wang, Y., Kinshuk y Chen, N.-S. (2014). Is FLIP enough? Or should we use the FLIPPED model instead? Computers \& Education, 79, 16-27. doi: 10.1016/j.compedu.2014.07.004

Goodwin, B. y Miller, K. (2013). Evidence on flipped classrooms is Still Coming In. Educational Leadership, 70 (6), 78-80. Recuperado de http://www.ascd.org/publications/

Herreid, C. y Schiller, N. (2013). Case studies and the flipped classroom. Journal of College Science Teaching, 42 (5), 62-66. Recuperado de https://www.aacu.org/

Imran, M. (2013). Increasing the interaction time in a lecture by integrating flipped classroom and just-in-time teaching concepts. Journal of Learning and Teaching, 7, 1-13. doi: 10.21100/compass.v4i7.84

@JOSÉ PALAZÓN-HERRERA. THE CONTENT OF THIS ARTICLE IS THE SOLE RESPONSIBILITY OF THE AUTHORS. THE REVISTA ELECTRÓNICA DE LEEME AND UNIVERSITAT DE VALĖNCIA ARE NOT LIABLE FOR ANY LEGAL ACTIONS THAT MAY ARISE INVOLVING THE ARTICLE'S CONTENT. REVISTA ELECTRÓNICA DE LEEME -LISTA ELECTRÓNICA EUROPEA DE MÚSICA EN LA EDUCACIÓN-HTTP://OJS.UV.ES/INDEX.PHP/LEEME/INDEX ISSN: 1575-9563 EDITORES: UNIVERSIDAD DE VALENCIA Y JESÚS TEJADA GIMÉNEZ. VISIBILIDAD DE ESTA REVISTA: SCOPUS, EMERGING SOURCES CITATION INDEX (CLARIVATE), EBSCO, CINDOC (CSIC), CITEFACTOR, COPAC, DIALNET, DICE (CSIC), DOAJ, E-REVISTAS (CSIC), EBSCO PREMIER, ERIH+, GALE CENGAGE LEARNING, IN-RECS, IRESIE, LATINDEX, MIAR, OCLC WORLDCAT, RESH, REDIB, RILM CORE JOURNALS, SUDOC, ULRICHS, ESTA REVISTA
INSTITUCIONAL DE REDIRIS-CONSEJO SUPERIOR DE INVESTIGACIONES CIENTIFICAS Y ES DE ACCESO LIBRE. CREATIVE COMMONS LICENSE 4.O BY 
Kellinger, J. J. (2012). The flipside: Concerns about the "New literacies" paths educators might take. The Educational Forum, 76 (4), 524-536. Recuperado de https://www.learntechlib.org/

Kemmis, S. (1988). Action research. En J. P. Keeves (coord.), Educational Reseach, Methodology, and Measurement. An International Handbook. Oxford: Pergamon.

Lomax, P. (1995). Action research for professional practice. British Journal of In-Service Education, 21 (1), 49-57.

Long, T., Logan, J. y Waugh, M. (2016). Students' Perceptions of the Value of Using Videos as a Pre-class Learning Experience in the Flipped Classroom. TechTrends, 60 (3), 242-252. doi: 10.1007/s11528-016-0045-4

Mattis, K. V. (2015). Flipped classroom versus traditional textbook instruction: Assessing accuracy and mental effort at different levels of mathematical complexity. Technology, Knowledge and Learning, 20 (2), 231-248. Recuperado de https://www.researchgate.net/

McMillan, J. H. y Schumacher, S. (2005). Investigación educativa. Madrid: Pearson Educación.

McNiff, J. y Whitehead, J. (2002). Action research: Principles and practice. Londres: Routledge.

Parra, F. J. y Gutiérrez, I. (2017). Implementación y análisis de una experiencia de flipped classroom en Educación Musical. Innoeduca. International Journal of Technology and Educational Innovation, 3 (1), 4-14. doi: 10.24310/innoeduca.2017.v3i1.1964

Seaman, G. y Gaines, N. (2013). Leveraging digital learning systems to flip classroom instruction. Journal of Modern Teacher Quarterly, 1, 25-27.

Turro, C., Mengod, R., Morales, J. C. y Busquets, J. (2016). Video is key for Flipped Learning: An experience at Universitat Politecnica de Valencia. Comunicación presentada en Workshop on Smart Environments and Analytics in Video-Based Learning (SE@VBL), Edinburgh, Scotland. Recuperado de https://www.semanticscholar.org/

@JOSÉ PALAZÓN-HERRERA. THE CONTENT OF THIS ARTICLE IS THE SOLE RESPONSIBILITY OF THE AUTHORS. THE REVISTA ELECTRÓNICA DE LEEME AND UNIVERSITAT DE VALĖNCIA ARE NOT LIABLE FOR ANY LEGAL ACTIONS THAT MAY ARISE INVOLVING THE ARTICLE'S CONTENT. REVISTA ELECTRÓNICA DE LEEME -LISTA ELECTRÓNICA EUROPEA DE MÚSICA EN LA EDUCACIÓN-HTTP://OJS.UV.ES/INDEX.PHP/LEEME/INDEX ISSN: 1575-9563 EDITORES: UNIVERSIDAD DE VALENCIA Y JESÚS TEJADA GIMÉNEZ. VISIBILIDAD DE ESTA REVISTA: SCOPUS, EMERGING SOURCES CITATION INDEX (CLARIVATE), EBSCO, CINDOC (CSIC), CITEFACTOR, COPAC, DIALNET, DICE (CSIC), DOAJ, E-REVISTAS (CSIC), EBSCO PREMIER, ERIH+, GALE CENGAGE LEARNING, IN-RECS, IRESIE, LATINDEX, MIAR, OCLC WORLDCAT, RESH, REDIB, RILM CORE JOURNALS, SUDOC, ULRICHS, ESTA REVISTA ESTÁ PUBLICADA CON EL APOYO INSTITUCIONAL DE REDIRIS-CONSEJO SUPERIOR DE INVESTIGACIONES CIENTIFICAS Y ES DE ACCESO LIBRE. CREATIVE COMMONS LICENSE 4.0 BY 